\title{
Relativistic Diskoseismology
}

\author{
Robert V. Wagoner \\ Department of Physics and Center for Space Science and Astrophysics, \\ Stanford University, Stanford, CA 94305-4060, USA
}

\begin{abstract}
We will summarize results of relativistic calculations of the eigenfunctions and eigenfrequencies of modes of oscillation trapped within the inner region of thin accretion disks by non-Newtonian gravitational properties of a black hole (or a compact, weakly-magnetized neutron star). The focus will be on the most observable, robust, and best-studied class: the analogue of (internal) gravity modes in stars. The frequencies of the corresponding peaks in the power spectrum of the resulting luminosity modulations depend almost entirely upon only the mass and angular momentum of the black hole. The frequency width also depends upon the viscosity parameter of the disk. Searches for such features are being carried out by the RXTE satellite.
\end{abstract}

\section{Introduction}

Presently, evidence for the existence of black holes is based almost entirely upon observations which indicate that a certain amount of mass is contained within a region of a certain radius. However, a black hole is a region of spacetime governed by the Kerr metric of general relativity. We are aware of only three proposals for the observation of black hole signatures that could be definitive. The first involves the effect of the Kerr metric on the polarization of radiation emitted near the black hole (Connors, Piran, \& Stark 1980). The second involves the frequency and time dependence of emission line profiles (e.g., Fabian et al. 1989). The third is what we shall describe below.

Our group has been investigating consequences of the realization [by Kato and Fukue (1980)] that general relativity can trap normal modes of oscillation near the inner edge of accretion disks around black holes. The strong gravitational fields that are required can also be produced by neutron stars that are sufficiently compact (requiring a soft equation of state) and weakly magnetized that there is a gap between the surface of the star and the innermost stable orbit of the accretion disk. Although we shall not explicitly consider such neutron stars here, the results obtained will also apply to them to first order in the dimensionless angular momentum parameter $a=c J / G M^{2}$, since their exterior metric is identical to that of a black hole to that order. It should be noted that $a<0.2$ for most models of rotating neutron stars.

These modes of oscillation provide a potentially powerful probe of both strong gravitational fields and the physics of accretion disks, since:

- They do not exist in Newtonian gravity 
- Their frequencies depend upon the angular momentum as well as the mass of the black hole

- The fractional frequency spread of each mode is proportional to $\alpha$, the viscosity parameter of the accretion disk.

\section{Adiabatic Oscillations}

Following exploratory calculations by Kato (1980), Okasaki et al. (1987), and Kato (1989), we also employed a modified Newtonian potential to calculate the adiabatic eigenfunctions and eigenvalues of the lowest acoustic $(p)$ modes (Nowak \& Wagoner 1991) and internal gravity (g) modes (Nowak \& Wagoner $1992,1993)$. This was extended to full general relativity by Perez (1993), who also included the corrugation $(c)$ modes studied by Kato $(1990,1993)$ and Ipser $(1994,1996)$. The g-modes have been analyzed more extensively by Perez, Silbergleit, Wagoner, \& Lehr (1997), and we shall also summarize their key results below.

We have applied the general relativistic perfect fluid perturbation formalism of Ipser and Lindblom (1992) to thin accretion disks in the Kerr metric. The radial component of the velocity of the fluid is neglected, which has little effect on the modes for these thin models. Neglecting the gravitational field of the disk (also a good approximation), the adiabatic oscillations of all physical quantities can be expressed in terms of a single scalar potential $\delta V(r, z)$ governed by a second-order partial differential equation. The stationary $(\partial / \partial t=0)$ and axisymmetric $(\partial / \partial \phi=0)$ unperturbed accretion disk is specified by the relativistic $\alpha$-disk model (Novikov and Thorne 1973, Page and Thorne 1974).

All fluid perturbations are of the form $f(r, z) \exp [i(\sigma t+m \phi)]$. With angular velocity $\Omega$, the comoving frequency is $\omega(r)=\sigma+m \Omega(r)$. It is sufficient to consider eigenfrequencies $\sigma<0$ and axial mode integers $m \geq 0$. Nonaxisymmetric modes $(m \neq 0)$ should produce relatively little luminosity modulation, unless the disk is viewed close to edge-on. In general, the vertical extent of the modes within the disk is restricted by the requirement that $|\omega|$ be greater than the buoyancy frequency. Numerical results have been obtained for accretion disks which are barotropic (e. g., isentropic) on scales of order their thickness, in which case this restriction is not operative since the buoyancy frequency vanishes.

The effective radial wavelengths are significantly smaller than $r$, allowing a WKB expansion of the problem and approximate separation of the governing equations. The key ingredient is the relativistic behavior of the radial epicyclic frequency $\kappa(r)$, shown in Figure 1 with other important orbital frequencies. As usual, $\kappa$ is the frequency of radial perturbations of circular orbits of a free particle, while $\Omega_{\perp}$ is the same for vertical perturbations.

The radial dependence of the fluid perturbations are governed by the WKB relation

$$
\frac{d^{2} W}{d r^{2}}+\frac{g_{r r}\left(U^{t}\right)^{2}}{c_{s}^{2}(r, 0)}\left[\Psi\left(\frac{\Omega_{\perp}}{\omega}\right)^{2}-1\right]\left(\kappa^{2}-\omega^{2}\right) W=0,
$$

where $g_{r r}$ is a Kerr metric component, $U^{t}$ is the four-velocity component $d t / d \tau$, $c_{s}(r, z)$ is the speed of sound, and $\Psi(r)$ is the slowly-varying separation function. The eigenfunction $W(r)$ is related to a radial derivative of the potential $\delta V$, and 

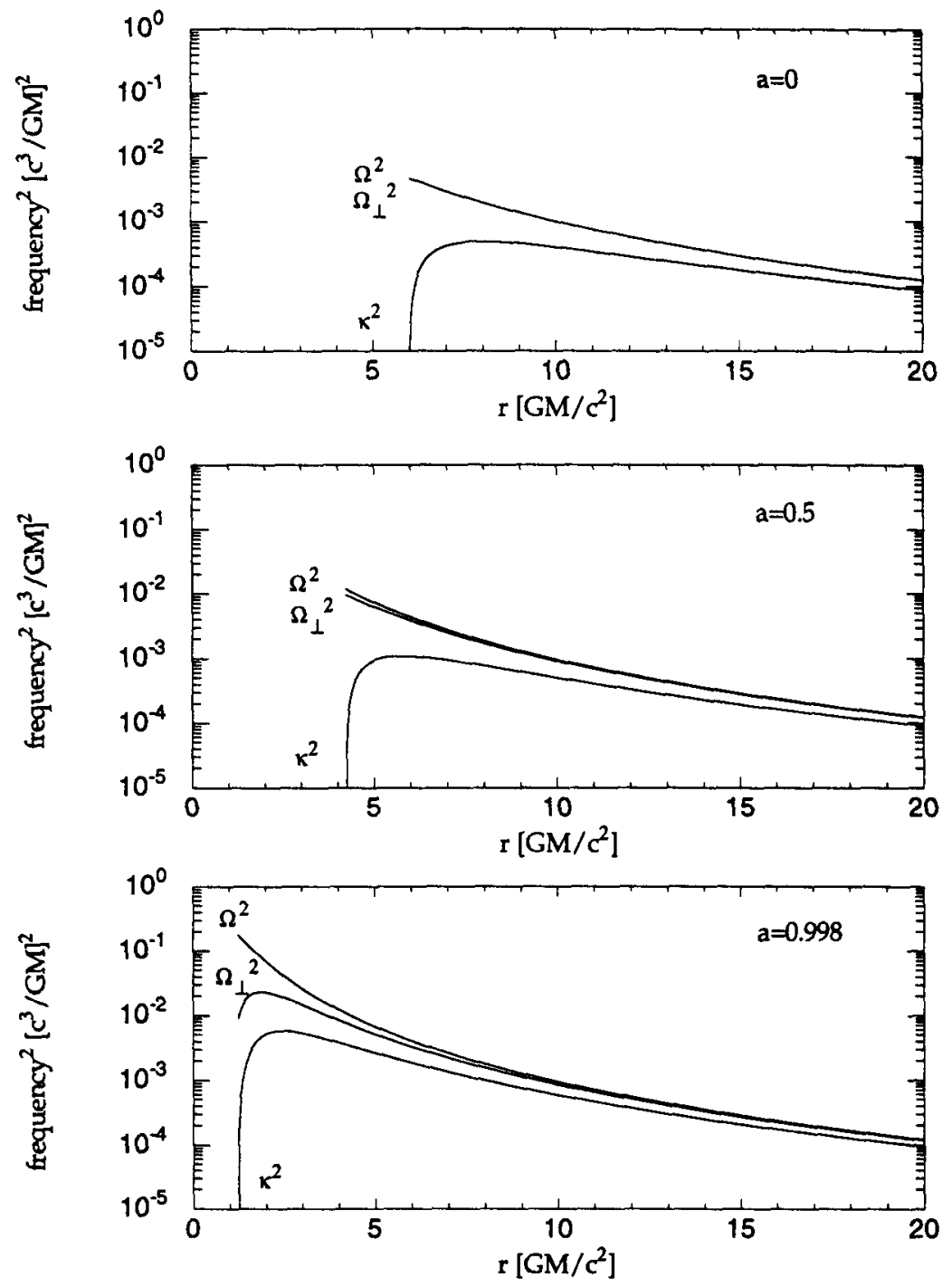

Figure 1. The radial dependence of the square of the fundamental free-particle frequencies that govern the modes of the disk: Keplerian $(\Omega)$, and radial $(\kappa)$ and vertical $\left(\Omega_{\perp}\right)$ epicyclic. Three values of the black hole angular momentum parameter $a=c J / G M^{2}$ are chosen. 
is proportional to the radial component of the fluid displacement. From equation (1), we can see where the modes are trapped $\left(W^{-1} d^{2} W / d r^{2}<0\right)$, as follows.

The $\mathrm{g}$-modes [defined by $\Psi\left(\Omega_{\perp} / \omega\right)^{2}>1$ ] are trapped where $\omega^{2}<\kappa^{2}$. This occurs between the radii $r_{-}(\sigma)$ and $r_{+}(\sigma)$, illustrated in Figure 2 and plotted in Figure 3. The lowest modes (fewest number of radial and vertical nodes in their eigenfunctions) have approximately vertical displacements, and have eigenfrequencies $|\sigma|$ which are close to the maximum possible, $\sigma_{m}=\kappa\left(r_{m}\right)+$ $m \Omega\left(r_{m}\right)$. Also shown in Figure 2 and plotted in Figure 4 is this radius $r_{m}$ to which $r_{-}$and $r_{+}$converge as the frequency is raised to this maximum.

The p-modes [defined by $\Psi\left(\Omega_{\perp} / \omega\right)^{2}<1$ ] are trapped where $\omega^{2}>\kappa^{2}$. The radial $(m=0) \mathrm{p}$-modes that are trapped within the inner radius of the disk $\left(r_{i}\right)$ and $r_{-}$have very little radial extent, and thus will produce negligible direct luminosity modulation, although they will modulate the accretion onto the black hole (or neutron star). The highest frequency modes that are trapped within $r_{+}$and the outer radius of the disk $\left(r_{0}\right)$ will modulate a significant fraction of the disk where the luminosity per unit radius is highest. However, since their wavelength $\lambda(r) \sim c_{s} / \omega$ will be relatively short, the net luminosity modulation will be reduced. We have not yet investigated this outer branch of $\mathrm{p}$-modes because of the uncertain physics and location of the outer radius.

The c-modes [defined by $\Psi\left(\Omega_{\perp} / \omega\right)^{2} \cong 1$ ] are typically nonradial $(m=1)$ incompressible (and therefore less modulating) waves that slowly precess around the angular momentum of the black hole. The ones that we have studied have a maximum frequency proportional to $a^{3}$, only becoming comparable to the gmode frequency $\sigma_{m}$ when $a \rightarrow a(\max ) \cong 0.998$. They also tend to exist very close to the inner edge of the disk.

The frequencies of all of these modes are proportional to $1 / M$, but their dependence on the angular momentum of the black hole is quite different (Perez 1993). However, we now turn our attention to the g-modes, which are the most robust and observable of those classes that we have investigated.

The dependence of the maximum $\mathrm{g}$-mode frequencies on the angular momentum of the black hole is illustrated in Figure 5. For instance, the lowest radial $(m=0) \mathrm{g}$-modes have a frequency $f=-\sigma / 2 \pi$ given by

$$
f=714\left(1-\epsilon_{n j}\right)\left(M_{\odot} / M\right) F(a) \mathrm{Hz}, \quad \epsilon_{n j} \approx\left(\frac{n+1 / 2}{j+\delta}\right) \frac{h}{r_{0}} .
$$

The properties of the accretion disk enter only through the small term $\epsilon_{n j}$, which involves the disk thickness $2 h\left(r_{0}\right)$ and the radial $(n)$ and vertical $(j)$ mode numbers, with $\delta \sim 1$. Typically $h\left(r_{0}\right) / r_{0} \sim 0.1 L / L_{E d d}$ for a radiation-pressure dominated optically thick disk region. $F(a)$ is the upper function plotted in Figure 5. From Figure 5, we also see that the higher axial modes have a somewhat different dependence upon the black hole angular momentum, which in principle would allow its determination as well as that of the mass.

The effective fractional width $\left[\Delta r(f) / r_{m}\right]$ of the lowest eigenfunction (outside of which it becomes evanescent) is shown in Figure 4. Although the approximate center of the eigenfunction (located at $r_{m}$ ) is close to the temperature maximum of the disk, its relatively small width appears to limit the luminosity modulation to at most a few percent. 

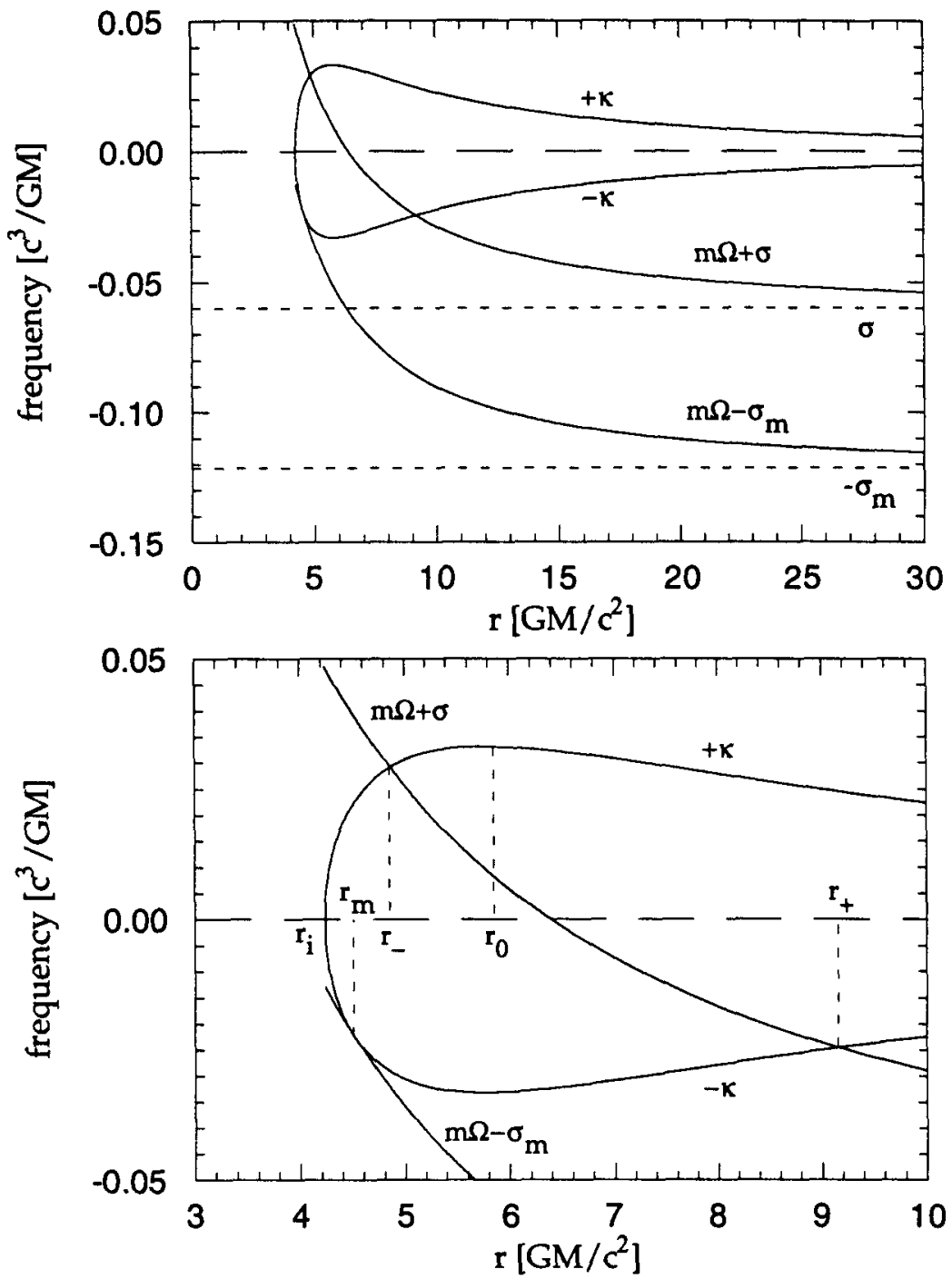

Figure 2. The functions which determine the radii $r_{-}(\sigma)$ and $r_{+}(\sigma)$ between which the eigenfunctions are concentrated. Also indicated is the maximum value $\sigma_{m}$ of the eigenfrequency $|\sigma|$, and the inner radius $r_{i}$ of the disk. For this plot, we choose $a=0.5$ and $m=1$ (with the case $m=0$ corresponding to use of the dashed horizontal lines). 

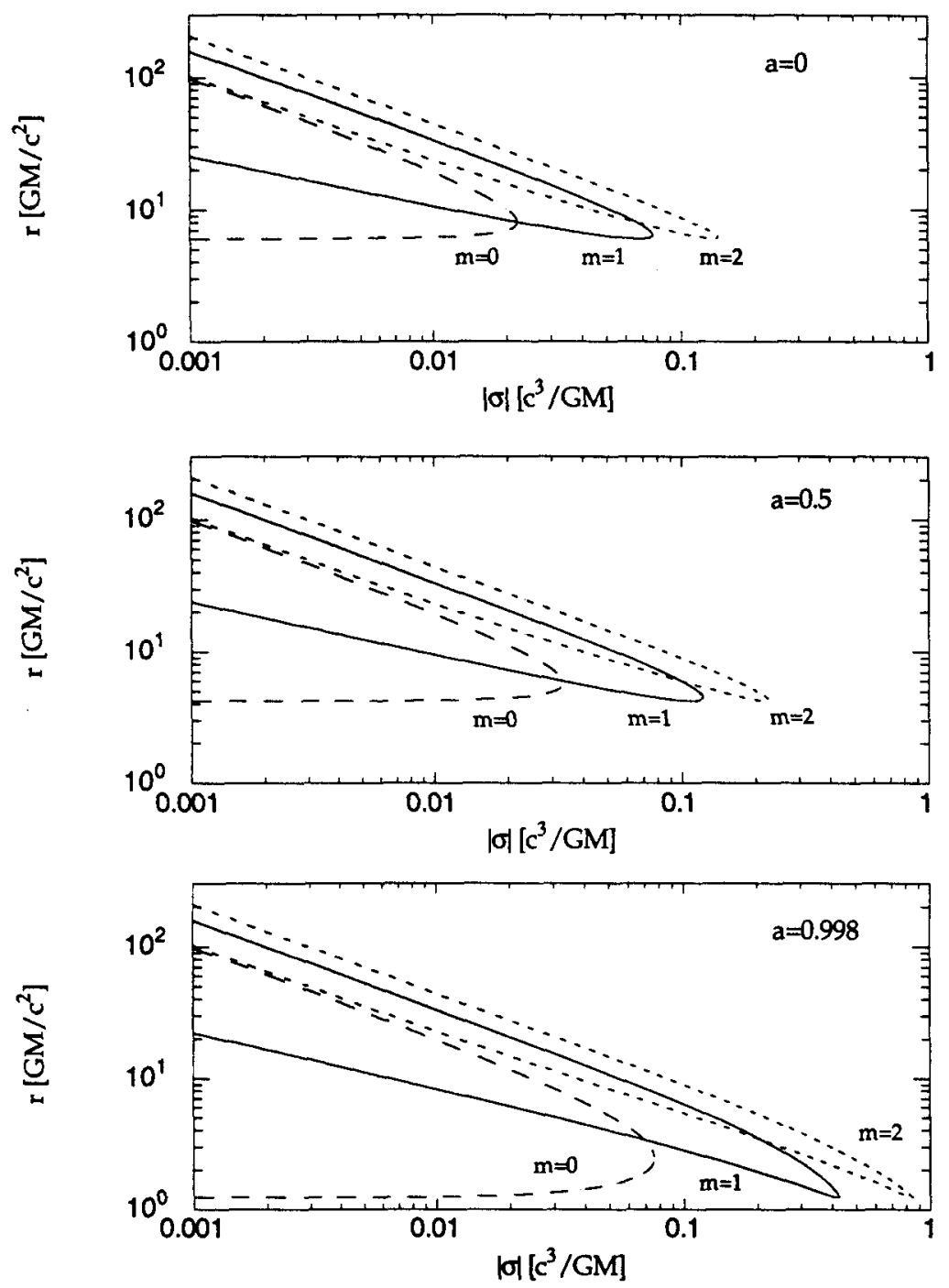

Figure 3. The radii between which a g-mode eigenfunction is concentrated is plotted versus its eigenfrequency. 

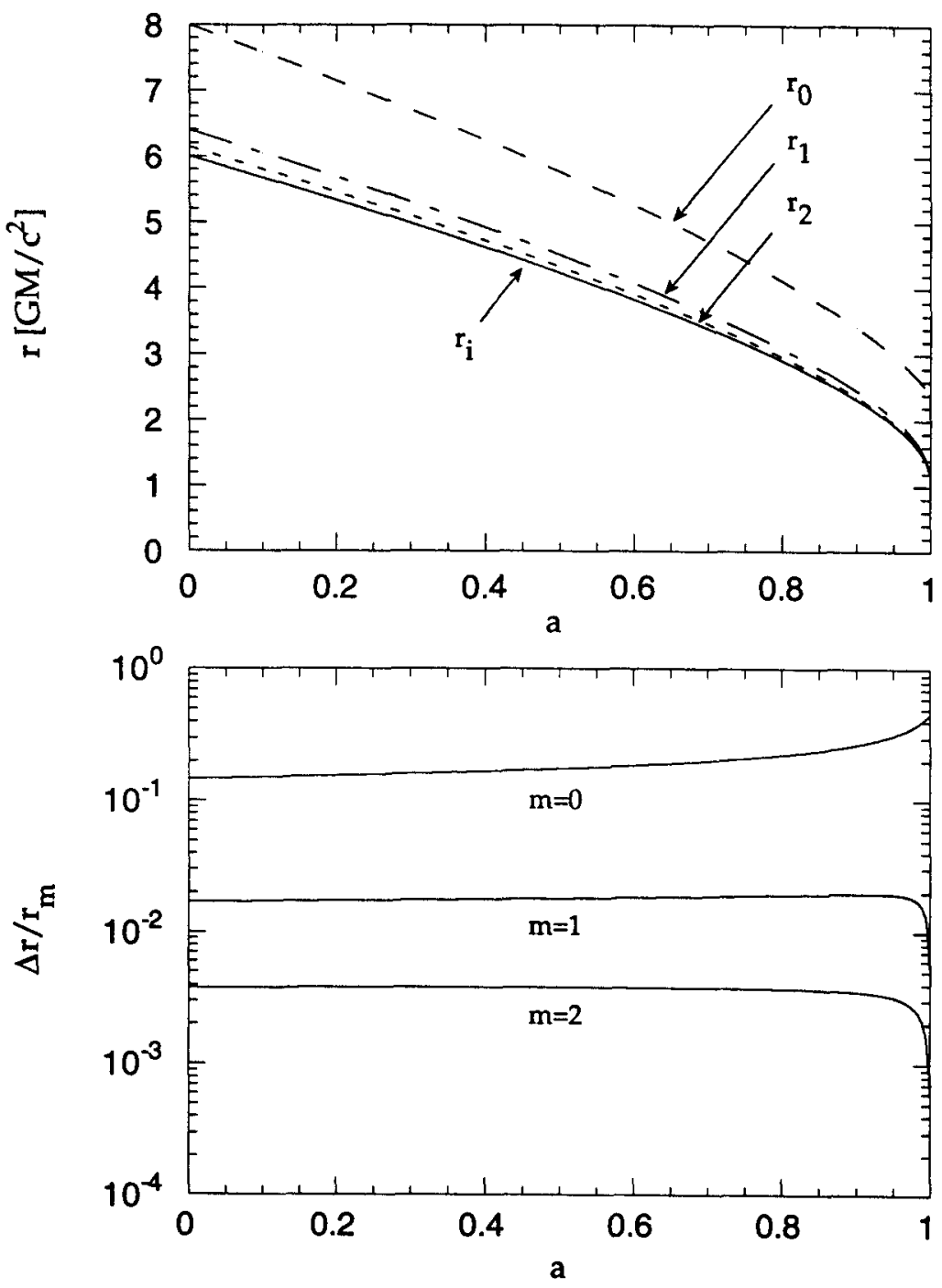

Figure 4. (a) The black hole angular momentum dependence of the radius $r_{m}$ to which $r_{-}$and $r_{+}$converge as $|\sigma| \rightarrow \sigma_{m}$. Also shown is the radius $r_{i}$ of the inner edge of the disk. (b) The dependence of the fractional effective width of the lowest eigenfunction, $\Delta r / r_{m}=$ $\left[r_{+}(\sigma)-r_{-}(\sigma)\right] / r_{m}$, on the angular momentum of the black hole. The same values of $m$ are chosen as in (a). The accretion disk model is specified by $\Gamma=4 / 3$, a locally isentropic equation of state, and speed of sound corresponding to a luminosity $L=0.1 L_{E d d}$ from a radiationdominated disk. 

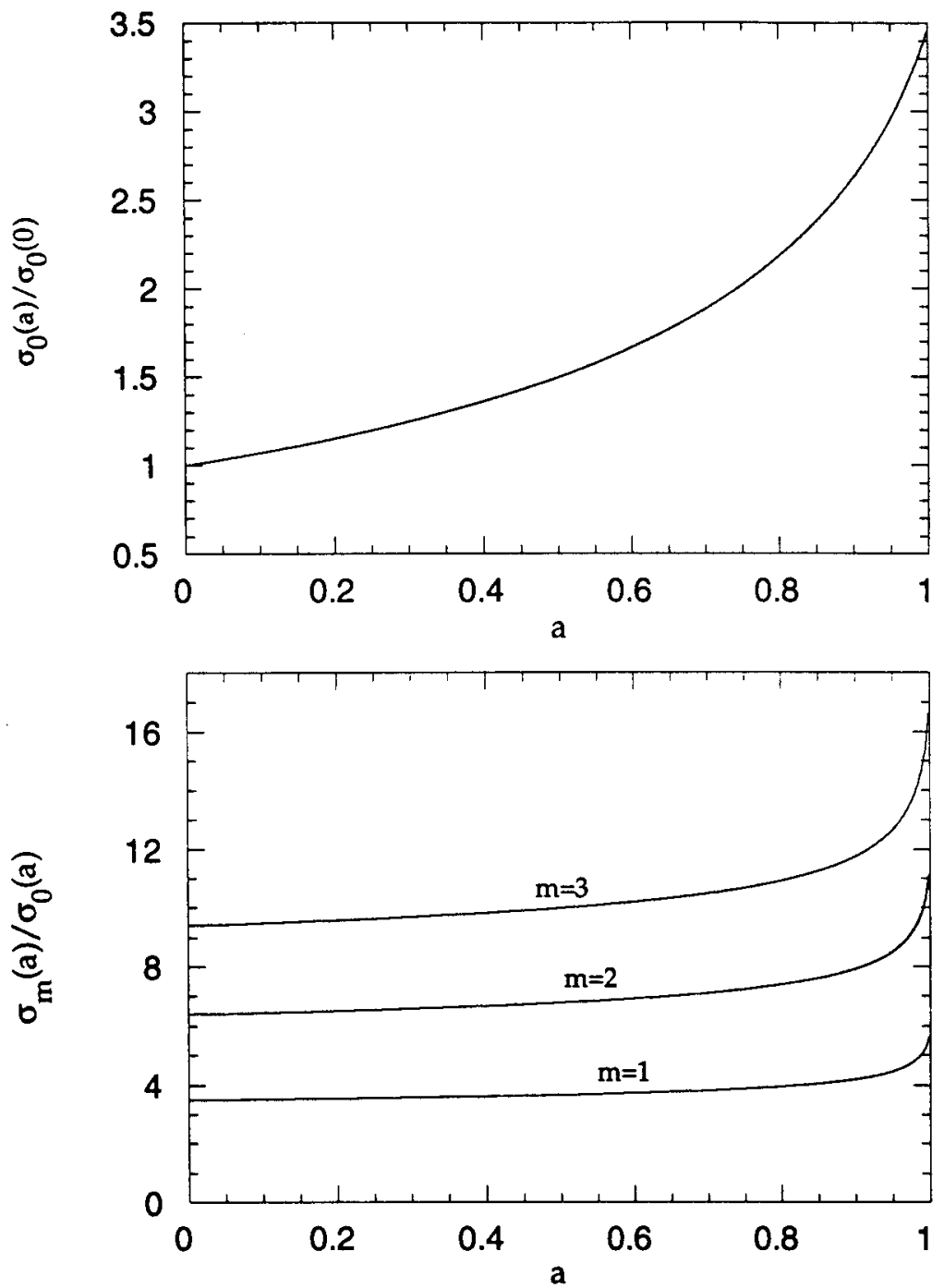

Figure 5. (a) The dependence of the maximum radial $(m=0)$ eigenfrequency on the black hole angular momentum parameter $a=$ $c J / G M^{2}$, relative to its value at $a=0$. (b) The ratio of the maximum eigenfrequency of some higher $m$ modes to that of the radial mode. 


\section{Frequency Width and Amplitude of the Oscillations}

Nowak and Wagoner (1992) have estimated the damping (or growth) rates of $p$ and $\mathrm{g}$-modes due to isotropic and anisotropic turbulent viscosity and gravitational radiation reaction. Although the gravitational radiation instability will cause the modes to grow for $M \gg 10^{8} M_{\odot}$, we have recently found that isotropic viscosity will always produce damping. With $j$ and $n$ of order the number of vertical and radial nodes in any particular eigenfunction, its corresponding quality factor $Q$ is given by

$$
1 / Q_{j n} \sim\left[j^{2}+(h / r) n^{2}\right] \alpha,
$$

where the viscosity parameter is thought to lie in the range $10^{-2}<\alpha<1$. However, the effective width $\Delta f$ of the $g$-mode feature will also be determined by what range of mode numbers $j$ and $n$ are sufficiently excited [with each mode broadened by $\left.(\Delta f / f)_{j n} \approx 1 / Q_{j n}\right]$.

An estimation of the amplitude of the oscillations, and the resulting fractional modulation of the disk luminosity, was also obtained by Nowak and Wagoner (1993). They found approximate equipartition between the energy of the lowest modes and the energy of the largest turbulent eddies [presumably driven by the Velikov-Chandrasekhar-Balbus-Hawley magnetic instability [for recent numerical results, see Stone et al. (1996)]. This produces maximum $g$-mode displacements of order the thickness of the disk, which should produce maximum luminosity modulations $\delta L_{\nu} / L_{\nu} \sim 10^{-2}$, if the photon frequency $\nu>h^{-1} k T(\max )$.

If turbulence drives these oscillations, it should also contribute to the fluctuation power in a broad range of frequencies. Nowak and Wagoner (1995) have computed the resulting power spectral density, and found that it could match that of the best-observed black hole candidate Nova Muscae (Miyamoto et al. 1993), but only at frequencies $f>10 \mathrm{~Hz}$. The power is predicted to drop rapidly $\left(\propto f^{-5}\right)$ above a frequency given approximately by equation (2).

\section{Observations}

These modes of oscillation should modulate the quasi-thermal emission from accretion disks in the ultraviolet for supermassive black holes and at soft $\mathrm{x}$-ray energies for stellar mass black holes.

R. Wagoner, M. Nowak, and W. Zhang have been granted target of opportunity time with the RXTE satellite to obtain power spectra (from 0.001 to 2000 $\mathrm{Hz}$ ) of the black hole candidate GX 339-4 and a transient such as GS 1124-68 (Nova Muscae), during a 'high quiet' state dominated by a thermal disk energy spectrum. This was chosen to reduce the possibility of demodulation of the disk emission by a halo. Simulations indicate that a $\mathrm{g}$-mode feature in the power spectrum with amplitude greater than $1 \%$ (for $Q \sim 3$ ) should be detectable with a few hours integration.

Recently, Morgan et al. (1996) have detected a feature at $f=67 \mathrm{~Hz}$ with $Q \approx 20$ in the black hole candidate GRS $1915+105$ with RXTE. What distinguishes this peak from others that have recently been detected at high frequencies is the fact that it did not change its frequency as the source luminosity varied. During the time that the feature was detectable, the source varied from 
1-2 Crab but the frequency changed by less than $3 \%$. This is clearly predicted by equation (2). The amplitude of the peak was greatest $(\sim 6 \%)$ at the highest $\mathrm{x}$-ray energies.

If this feature is produced by a g-mode oscillation, equation (2) predicts a black hole mass of $10.6 M_{\odot}$ if it is nonrotating to $36.3 M_{\odot}$ if it is maximally rotating. Other aspects of this identification are explored by Nowak et al. (1996).

Acknowledgments. Most of the recent results reported here were obtained in collaboration with Dana E. Lehr, Michael A. Nowak, Christopher A. Perez, and Alexander S. Silbergleit. The research was supported in part by NASA through ATP grant NAG 5-3102 to R.V.W., NASA grant NAS8-39225 to Gravity Probe B, and a National Defense Science and Engineering Graduate Fellowship to D.E.L.

\section{References}

Connors, P.A., Piran, T. \& Stark, R.F. 1980, ApJ, 235, 224

Fabian, A.C., Rees, M.J., Stella, L., \& White, N.E. 1989, MNRAS, 238, 729

Ipser, J.R. 1994, ApJ, 435, 767

Ipser, J.R. 1996, ApJ, 458, 508

Ipser, J.R., \& Lindblom, L. 1992, ApJ, 389, 392

Kato, S. 1989, PASJ, 41, 745

Kato, S. 1990, PASJ, 42, 99

Kato, S. 1993, PASJ, 45, 219

Kato, S., \& Fukue, J. 1980, PASJ, 32, 377

Miyamoto, S., et al. 1993, ApJ, 403, L39

Morgan, E., et al. 1996, IAUC 6392.

Nowak, M.A., \& Wagoner, R.V. 1991, ApJ, 378, 656

Nowak, M.A., \& Wagoner, R.V. 1992, ApJ, 393, 697

Nowak, M.A., \& Wagoner, R.V. 1993, ApJ, 418, 187

Nowak, M.A., \& Wagoner, R.V. 1995, MNRAS, 274, 37

Nowak, M.A., Wagoner, R.V., Begelman, M.C., \& Lehr, D.E. 1996, ApJ, submitted

Novikov, I.D., \& Thorne, K.S. 1973, in Black Holes, ed. C. DeWitt \& B.S. DeWitt (New York: Gordon \& Breach), p. 343

Okazaki, A.T., Kato, S., \& Fukue, J. 1987, PASJ, 39, 457

Page, D.N., \& Thorne, K.S. 1974, ApJ, 191, 499

Perez, C.A. 1993, Ph. D. thesis, Stanford University

Perez, C.A., Silbergleit, A.S., Wagoner, R.V., \& Lehr, D.E. 1997, ApJ, in press (vol. 476, Feb. 20)

Stone, J.M., Hawley, J.F., Gammie, C.F., \& Balbus, S.A. 1996, ApJ, 463, 656 


\section{Discussion}

R. Nelson: Rotating fluids can support negative energy modes. In the presence of dissipation, these modes will grow. This is the so-called Friedmann-Schuty instability in rotating General Relativistic stars, although Relativity is not required - only dissipation. Do you all find any unstable negative energy modes in your disks?

R. Wagoner: Yes, we do have negative energy modes, for axial quantum numbers $m>0$. We [Nowak and Wagoner, ApJ 393, 697 (1992)] have calculated the growth rate of $m=2$ modes due to the CFS gravitational radiation instability, and find that it is less than that due to $\alpha$-viscosity for all black hole masses $M \leq 10^{9} \mathrm{M}_{\odot}$.

R. Soria: Have you tried to extend your analysis of eigenfrequencies to the case of naked singularities $(a>1)$ ? Could such a study provide an observational clue to distinguish between $\mathrm{BH}$ and naked singularity?

R. Wagoner: Our analysis can be generalised to any time-independent, axiallysymmetric spacetime in which a disk of matter rotates in circular, stable orbits. The eigenfrequencies of the perturbations do strongly probe the structure of the gravitational field. 